\section{The postcholecystectomy syndrome: a review of etiology and current approaches to management}

\author{
Robert D. Kung, Amanda W. Cai, \\ Jason M. Brown, Anthony M. Gamboa, \\ Qiang Cai \\ Division of Digestive Diseases, Emory \\ University, School of Medicine, Atlanta, \\ GA, USA
}

\begin{abstract}
Postcholecystectomy syndrome (PCS) comprises a heterogeneous group of symptoms and disorders in patients who have previously undergone cholecystectomy. While it is relatively uncommon, it is defined by chronic recurring pain, often with no clear source. Recent studies suggest its pathogenesis depends on different factors, but it remains poorly understood and complex to treat. Here, we present a brief overview of this syndrome, review recent literature regarding its etiology, and present a systematic approach to diagnosis and management.
\end{abstract}

\section{Introduction}

Cholecystectomy is one of the most commonly performed surgical procedures and is the standard of care in treating symptomatic gallstones. ${ }^{1-4}$ Up to a third of patients undergoing cholecystectomy will develop recurrent and persistent abdominal pain weeks to years after surgery. ${ }^{5-6}$ In the majority of patients, symptoms are mild and short lived, but $2-5 \%$ will continue to have frequent debilitating pain, a condition referred to as the postcholecystectomy syndrome (PCS). ${ }^{5}$ Rather than being a single disorder, this term encompasses a widely varying group of disorders, including extrabiliary, organic biliary, and functional biliary diseases, considered true PCS (Table 1). In many cases, no source of PCS can be identified, and symptoms remain unexplained and challenging to treat. This article is a review of the recent literature regarding the different etiologies of PCS, and current diagnostic and therapeutic approaches to its management.

\section{Extrabiliary etiology}

The most common causes of PCS may be extrabiliary diseases, an umbrella classification including gastroesophageal reflux disease, gastritis, peptic ulcer disease, irritable bowel syndrome, chronic pancreatitis, and malignancy. ${ }^{5,7}$ Lasson et al. followed 65 patients initially diagnosed with PCS and found $48 \%$ with identifiable extrabiliary diseases during 4-13 years of follow up. ${ }^{8}$ Similarly, Filip et al. found 27 of 80 patients (34\%) evaluated for PCS actually had non-biliary symptoms. ${ }^{9}$ Others cite gastroparesis, costchondritis, fibromyalgia, and chronic narcotic use as common causes for PCS pain. ${ }^{10}$ Thus, the first step in evaluating patients with PCS must be a careful history and evaluation to exclude these common and often treatable disorders. Given the number of non-organic pain disorders included in the differential, referral to a pain specialist may also be appropriate.

\section{Organic biliary etiology}

New biliary disorders may develop after cholecystectomy, and these must be ruled out through standard laboratory and radiological assessments. Common bile duct stones and cholangiocarcinoma can arise independently of cholecystectomy. Obstructive symptoms require relief of the obstruction with prompt endoscope retrograde cholangiopancreatography (ERCP) or surgical intervention. Malignancy is associated with older age and later presentation. Filip et al. recently published a cohort study demonstrating endoscopic ultrasound was $96.2 \%$ sensitive and $88.9 \%$ specific for identifying pancreaticobiliary diseases. ${ }^{9}$ This approach helped define which patients warranted subsequent ERCP, reducing both the number of ERCPs by $51 \%$ and the procedure related morbidity associated with this procedure. Surgically related complications such as strictures and bile duct injuries have been described extensively elsewhere and are not the focus of this review. ${ }^{11}$

\section{Functional biliary etiology: true postcholecystectomy syndrome}

\section{Sphincter of Oddi dysfunction}

When basic evaluation has ruled out both extrabiliary disorders and gross biliary obstruction, sphincter of Oddi dysfunction (SOD) is believed to be the most frequently identifiable cause of PCS. While the estimated incidence is less than $1 \%$ of all cholecystectomized patients, SOD may account for $14 \%$ of pain from PCS. ${ }^{12}$ Despite the prominence of SOD in the differential diagnosis of PCS, the pathogenesis of SOD remains poorly understood. Two mechanisms have been proposed: sphincter of Oddi stenosis refers to a structural abnormality resulting from inflammation and scarring of the sphincter. Sphincter of Oddi basal pressures, which can be measured by manometry (SOM) during ERCP, tend to be above $40 \mathrm{mmHg}$, and do not change after pharmacological challenge. The second is sphincter
Correspondence: Qiang Cai, Division of Digestive Diseases, Emory University School of Medicine, 1365 Clifton Road, B1262 Atlanta, GA 30322, USA. Tel: +1.404.778.4857 - Fax: +1.404.778.2578.

E-mail: qcai@emory.edu

Key words: postcholecystectomy syndrome, cholecystectomy, etiology.

Received for publication: 9 June 2011. Accepted for publication: 29 August 2011.

This work is licensed under a Creative Commons Attribution NonCommercial 3.0 License (CC BYNC 3.0).

@C Copyright Robert D. Kung et al., 2012

Licensee PAGEPress, Italy

Gastroenterology Insights 2012; 4:e1

doi:10.4081/gi.2012.el

of Oddi dyskinesia, referring to a functional abnormality of the sphincter leading to intermittent obstruction, perhaps due to still unknown neuro-hormonal disturbances. ${ }^{13}$ Stenosis is thought to occur in type $1 \mathrm{SOD}$, inferred from its predictable response to sphincterotomy, whereas dyskinesia is thought to occur in type 2 and type $3 \mathrm{SOD}$, which are less understood.

Geenan and Hogan were the first to demonstrate relief of pain after endoscopic biliary sphincterotomy (EBS) in 91\% (10 of 11) of cholecystectomized patients with elevated sphincter of Oddi (SO) pressures at 12-month follow up, but no benefit in patients with normal sphincter pressures. ${ }^{14}$ By contrast, two recent studies have found only $18-43 \%$ of patients with definite SOD (i.e. proven by SOM) had complete resolution of symptoms at 12-18 months after EBS. ${ }^{14-15}$ This suggests a pathogenesis which is more complex than sphincter dysmotility alone. Several studies have implicated the development of visceral hyperalgesia and somatosensory hypersensitivity as a source of PCS which has been overlooked. Desautels et al. reproduced pain symptoms in type III SOD patients by distending the duodenum. ${ }^{16}$ Kuruscai et al. demonstrated increased hypersensitivity of peripheral nociceptive nerve fibers in the right upper quadrant of the abdomen. Less electrical stimuli were needed to illicit sensation in SOD patients when compared to asymptomatic cholecystectomized patients, symptomatic non-SOD patients, and healthy volunteers. It is thought that both peripheral nerve fibers and central nerve fibers at the level of the spine are hypersensitized, lowering the pain threshold, similar to the pathogenesis of other functional pain disorders, such as irritable bowel syndrome. ${ }^{17}$ Pain associated with injection of contrast or saline during ERCP has been common- 
ly reported, but its significance is unknown.18 Linder et al. quantitatively measured symptom improvement in patients with proven SOD who underwent EBS compared to those with suspected SOD with normal SOM. ${ }^{19}$ At 18 -months follow-up, the SOD/EBS group was more likely to express symptom improvement, but both groups almost uniformly continued to experience pain, consistent with chronic pain disorders and suggesting a multifactorial etiology.

SOD has been historically classified into 3 types according to the modified Milwaukee Criteria, based on the presence or absence of 3 criteria: biliary type abdominal pain; elevation of serum transaminases, alkaline phosphatase, or bilirubin to 1.5 times the upper limit of normal with normalization between attacks; and bile duct dilatation of more than $12 \mathrm{~mm}$ on imaging. ${ }^{14}$ Type I is defined as the presence of all 3 criteria, Type II as pain plus one other criteria, and Type III as pain alone. This classification is useful in identifying the subset of patients with Type I SOD, who have a greater than $90 \%$ chance of symptomatic improvement from EBS regardless of the results of manometry (Table 2)..$^{20-24}$ This consistent response suggests that the underlying pathophysiology of Type I SOD may be SO stenosis; however, no studies have proven this association. It is now common practice to bypass SOM and proceed straight to EBS in this subset of patients. ${ }^{23-24}$ The management of Type II and Type III SOD is less defined. While ERCP is beneficial for Type I, patients with Type II and III are less likely to have elevated sphincter pressures and respond to EBS. This is particularly true for patients with Type III SOD, whose response to EBS approaches only $37 \%$ even after abnormal SOM. ${ }^{25}$ Given the high risk of ERCPrelated pancreatitis, which is 2-9\% in unselected series and as high as $10-30 \%$ in cases of suspected SOD, ${ }^{26-27}$ the role of ERCP and SOM in this subset of patients is still a subject of debate. In a recent cohort study, 54\% of patients with suspected Type II or III SOD underwent ERCP and SOM and were found to have normal SOM, and $12 \%$ developed procedure-related pancreatitis. ${ }^{28}$ Using a symptom scoring survey, Madacsy et al. showed that this SOD negative PCS group was more likely to complain of atypical abdominal pain; typical abdominal pain experienced by patients with SOD is steady, lasts 15-30 minutes, localized to the epigastrum and right upper quadrant (RUQ), and is exacerbated by fatty foods. This delineation between typical and atyp- ical biliary pain follows the Rome III consensus criteria for diagnosing functional biliary pain, although further studies are needed to prove its utility in managing these patients. ${ }^{29}$ Other mechanisms such as visceral hyperalgesia are more likely to be playing a role in this group of PCS patients, many of whom are likely mislabeled as having SOD. ${ }^{16}$

Given the potential morbidity, cost, and lack of availability of SOM, non-invasive strategies to evaluate SOD are needed. The morphineprostigmin test (Nardi test) was one of the earliest methods developed, but has very low sensitivity and specificity for predicting the presence of SOD. ${ }^{30}$ Initial studies demonstrated that quantitative hepatobiliary scintigraphy (QHBS), measuring the hepatic hilum to duodenal transit time of radio-labeled tracer, correlated well with elevated SO basal pressures by SOM, the gold standard, had a sensitivity of $83 \%$ and specifici- ty of $100 \%{ }^{28,31-33}$ However, later studies found only a $13 \%$ sensitivity despite $95 \%$ specificity. ${ }^{34,35}$ These disparate results have raised questions regarding the reproducibility of scintigraphic results. The addition of morphine provocation appeared to increase the sensitivity to $83 \%$, and was associated with $81 \%$ specificity. ${ }^{36}$ Many recent trials have studied how well secretin-stimulated magnetic resonance cholangiopancreatography (ss-MRCP) predicts the results of SOM. It is to be noted that these studies included patients with biliary and pancreatic type pain, idiopathic pancreatitis, as well those with and without prior cholecystectomy. Prolonged increase in pancreatic duct diameter after injection of secretin, which relaxes the SO, had previously been shown to correlate well with ERCP diagnosis of papillary stenosis, but had not been correlated with SOM. ${ }^{37}$ Overall, the results have been disappointing (Table 3). Aisen et al.

Table 1. Etiologies of postcholecystectomy syndrome.

\begin{tabular}{lll} 
Exxtrabiliary & Organic biliary & Functional Biliary \\
GERD & Choledocholithiasis & Sphincter of Oddi dysfunction \\
Gastritis & Chronic pancreatitis & Biliary microlithiasis \\
\hline Gastroparesis & Pancreatic cancer & Visceral hypersensitivity/hyperalgesia \\
& & Somatosensory hyperalgesia \\
\hline Irritable bowel syndrome & Cholangiocarcinoma & $\begin{array}{l}\text { Remnant cystic duct } \\
\end{array}$ \\
& & Retained calculi \\
& Neuroma \\
& Hyperalgesia
\end{tabular}

Peptic ulcer disease

Nonulcer dyspepsia

Duodenal motility abnormality

Narcotic bowel

Duodenal diverticula

Malignancy

Abdominal wall pain (associated with Carnett's sign)

Chest wall tenderness

Costochondritis

Fibromylagia

Table 2. Response to endoscopic biliary sphincterotomy in sphincter of dysfunction by Milwaukee classification.

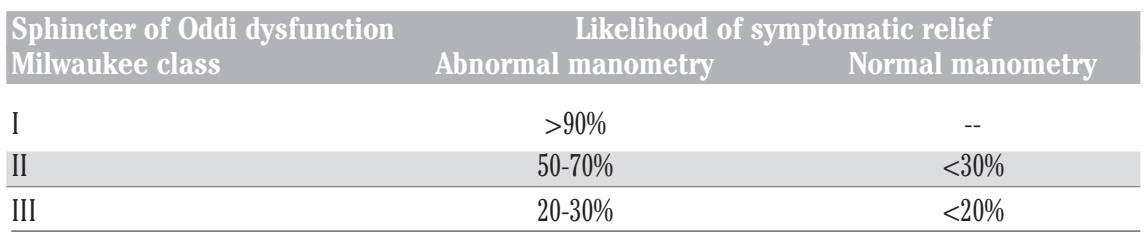

Milwaukee class: presence of RUQ pain, transaminitis $>1.5$ ULN, bile duct $>10 \mathrm{~mm}$ on imaging. Class I: all 3 criteria; Class II: pain +1 criterium; Class III: pain alone.

Table 3. Sensitivity, specificity, positive predictive value, negative predictive value of ss- secretin stimulated magnetic resonance cholangiopancreatography in the diagnosis of sphincter of Oddi dysfunction; PPV, positive predictive value; NPV, negative predictive value.

\begin{tabular}{|c|c|c|c|c|c|c|c|}
\hline Trial & \# of patients & \# of pts with SOD & Prior cholecystectomy (\%) & Sensitivity & Specificity & PPV & NPV \\
\hline $\begin{array}{l}\text { Pereira, } 2007 \\
\text { type II } \\
\text { type II+III }\end{array}$ & $\begin{array}{c}47 \\
-\end{array}$ & 27 & $\begin{array}{c}57 \\
-\end{array}$ & $\begin{array}{c}62.5 \\
37\end{array}$ & $\begin{array}{l}85 \\
85\end{array}$ & $\begin{array}{l}83 \\
83\end{array}$ & $\begin{array}{l}65 \\
39\end{array}$ \\
\hline Aisen, 2008 & 30 & 20 & 47 & 10 & 90 & 67 & 33 \\
\hline
\end{tabular}

ss-MRCP, secretin stimulated magnetic resonance cholangiopancreatography; SOD, sphincter of Oddi dysfunction; PPV, positive predictive value; NPV, negative predictive value. 
found that pancreatic duct measurement during ss-MRCP was no different in those with normal and elevated sphincter pressures, and fails to predict SOD. ${ }^{38}$ Pereira $e t$ $a l$. found that ss-MRCP had only $63 \%$ sensitivity but $85 \%$ specificity for diagnosing manometrically proven Type 2 SOD, with corresponding pain relief following BSE. The combined sensitivity in predicting both Types 2 and 3 SOD fell to 37\%. The authors concluded that ss-MRCP may have a role in selecting patients with suspected Type II SOD who might benefit from sphincterotomy. ${ }^{39}$ In the absence of better diagnostic tools, a recent trial evaluated a strategy to manage SOD without SOM.40 Those with Type I SOD and Type II SOD with dilated bile ducts on imaging were given the choice of medical therapy with or without sphincterotomy, with the possibility of trying the alternative if symptoms persisted beyond 3-6 months. Those Type II SOD patients without dilated bile ducts and all Type III SOD were managed with medical therapy alone. The proportion of participants reporting symptomatic improvement was similar to that previously reported in the literature, however, $55 \%$ of those enrolled had intact gallbladders.

There is insufficient data to determine the existence of SOD in the presence of an intact gallbladder, since few will be offered SOM prior to cholecystectomy, meaning that the conclusions of this study cannot be applied to those with PCS.

\section{Biliary microlithiasis}

Increasing evidence suggests biliary microlithiasis (BM) is another important cause of PCS. Bile crystals, composed of cholesterol monohydrate crystals (CMC) and calcium bilirubinate granules (CBG), are the precursors to gallstones. By definition, microlithiasis cannot be directly visualized by conventional trans-abdominal ultrasound or CT. Bile from duodenal or bile duct aspirates can be examined for the presence of such microlithiasis. They are considered positive if 3 or more crystals are present in a high power view in a polarized microscope. Microlithiasis has already been identified as a cause of recurrent idiopathic acute pancreatitis in patients with an intact gallbladder, presumably due to transient obstruction of the ampulla or pancreatic duct. ${ }^{41,42}$ Microlithiasis has also been identified in the bile of cholecystectomized patients with recurrent abdominal pain, although what role they may play in PCS is still not clear. $^{43,44}$ In a recent randomized trial, $10 \%$ of PCS patients had microlithiasis identified in their bile. ${ }^{45}$ Patients were randomized to receive ursodeoxycholic acid (urso) or placebo for six months. The results demonstrated statistically significant improvement or resolution of symptoms in the urso group compared to the placebo group. In the second phase of the study, the control group switched to urso for six months, and the improvement in symptoms was also statistically significant. This positive response to urso strongly suggests BM as a cause of PCS pain.

A causal relationship between biliary microlithiasis and SOD has been debated. Elmi et al. conducted a retrospective study which reviewed 17 Type I SOD patients, who underwent ERCP/SOM and EBS with sweeping of the common bile duct with a basket for stones. Nine of 17 (53\%) patients had concurrent microlithiasis in their bile, and all responded similarly well to EBS. ${ }^{13}$ This study speculated that the passing of stones leads to fibrosis and scarring of the ampulla of vater, likely to be part of the pathogenesis of Type 1 SOD. An earlier and larger prospective study by Quallich et al. had found only a $5 \%$ prevalence of microlithiasis in 60 patients with Type II and Type III SOD with both normal and abnormal manometry, though these results did not meet statistical significance. ${ }^{44}$ It is notable that bile sampling protocols were different between the studies: the former swept the common bile duct with a basket, while the latter aspirated from the common bile duct during ERCP. These differing conclusions may support the observation that Type 1 SOD is pathologically different from Types II and III SOD, with $\mathrm{BM}$ being the missing link.

Cystic duct remnant: retained calculi and neuroma

The cystic duct remnant left behind after cholestectomy has been recognized for many years to be a potential source of PCS pain. A randomized and blinded trial comparing complete removal of the cystic duct to conventional cholecystectomy found that $85 \%$ of patients were pain free over an 8-year follow-up period compared to $30 \%$ in the control group. ${ }^{46}$ Whether or not the cystic duct itself is painful is debatable, but both the presence of retained calculi and the development of traumatic neuroma have been identified as the underlying pathology in multiple studies. 47,48

By definition, a remnant cystic duct is more than $1 \mathrm{~cm}$ long and believed to be associated with a higher risk of retained calculi. Though the incidence is unknown, it appears to be growing in the laparoscopic era where higher rates of bile duct and vascular injuries encourage surgeons to leave behind a longer cystic duct or even part of the gall bladder. Walsh et al. reported patients developing recurrent biliary type pain 14 months to 20 years after either laparoscopic or open cholecystectomy, and 6 out of 7 patients were found to have retained cystic duct calculi on ERCP. ${ }^{47}$ Symptoms were relieved nearly a year after surgical resection of the remnant, extracorporeal shockwave lithotripsy (ESWL), or endoscopic biliary holmium laser lithotripsy. Palanivelu $e t$ al. retrospectively analyzed 9,590 patients undergoing laparoscopic cholecystectomy, finding an increased incidence of $4 \%$ amongst the group who underwent laparoscopic subtotal cholecystectomy. ${ }^{49}$ All underwent laparoscopic excision of the remnant duct and were pain free and asymptomatic at 3 -months follow up. In that series, ultrasound (US) identified these retained calculi with $60 \%$ accuracy, while MRCP was $92 \%$ accurate. These findings are consistent with earlier series that have highlighted MRCP as the test of choice in this setting. ${ }^{50}$ Recommendations on preventing remnant duct calculi at the time of initial surgery have been described: milking of the cystic duct before clipping it and routine use of intraoperative cholangiogram have been proposed. Adoption of these techniques into routine practice would likely reduce the incidence of this cause of PCS.

Cystic duct remnant neuromas are a rare cause of PCS pain. The nerves innervating the gall bladder are transected during cholecystectomy and can result in nerve hypertrophy with subsequent hyperalgesia or biliary stricture. This concept was challenged early on by an autopsy series demonstrating the presence of neuromas as common in asymptomatic cholecystectomized patients, suggesting these are normal post-operative changes and may not account for PCS pain. ${ }^{51}$ However, case series have reported pain from neuromas without biliary stricture; furthermore, others have demonstrated patients with obstructive jaundice due to biliary strictures arising from the neuromas. ${ }^{52,53}$

One case series reported that neuromas can cause pain without stricture formation by demonstrating temporary relief of pain after endoscopic ultrasound (EUS) guided injection of bupivicaine into the porta hepatis. ${ }^{48}$ All patients then underwent remnant duct resection with identification of the neuroma on pathology and significant pain relief, though recurrence months later was common. Whether or not the size, location, or other characteristics of the neuroma are associated with the presence and severity of symptoms remain unknown.

\section{Diagnostic and therapeutic approach}

There are currently no widely accepted guidelines for evaluating PCS, as PCS com- 
prises many heterogeneous disorders. With recent suggestions of a diverse and complex pathogenesis, clinicians should employ a systematic approach to diagnose and treat a range of potential etiologies (Figure 1).

The first step in the evaluation of a PCS patient should be a thorough history and physical examination to rule out common treatable conditions which may have been previously overlooked. Assessment for $H$. pylori infection, peptic ulcer disease, gastroparesis, amongst other disorders, should be initiated in the right clinical setting. Signs of extrahepatic obstruction warrant prompt evaluation with liver function tests and ERCP. EUS may be employed to verify absence of obstruction in patients with low suspicion for it.

The workup for true PCS should begin with differentiating functional pain from biliary pain, and Rome III criteria may be employed in this process. Having excluded a functional etiology, practitioners should evaluate patients for SOD. We propose re-labeling Type I SOD as definite SOD and Types II and III as suspected $S O D$ to avoid mislabeling many with no underlying SOD. Patients with definite SOD can be diagnosed with Modified Milwaukee Criteria with liver function tests and radiography, and should undergo ERCP and EBS. Patients with suspected SOD should first undergo non-invasive evaluations, though strategies have not been validated. Biliary scintigraphy has excellent specificity for predicting abnormal SOM, but a negative test does not rule out SOD. MRCP and EUS can be employed to diagnose pathology involving the cystic duct, such as retained stone. If the workup is negative, a multidisciplinary approach with pain management and psychiatry may be appropriate. SOM should be performed on patients with Type II SOD, but generally avoided with Type III SOD due to its limited utility and high complication rate.

Few studies have looked at the long-term efficacy of medical therapy for PCS or SOD. An empirical trial lasting several months of ursodeoxycholic acid may be reasonable if patients are not candidates for endoscopy, given the possibility of biliary microlithiasis in cholecystectomized patients. Its low cost and well tolerated side effect profile make this a particularly attractive option even without performing bile analysis. Two small double-blind, placebo controlled, crossover studies have shown that nifedipine can reduce the severity and frequency of pain, and need for analgesics compared to the placebo group. ${ }^{54,56}$ Nitrates have been shown to reduce the basal sphincter pressure in asymptomatic volunteers and symptomatic patients with SOD. Octreotide has also been shown to reduce basal sphincter pressures in manometrically proven SOD in a prospective randomized placebo controlled trial. ${ }^{56}$ Nitroglycerine, spasmolytics, opiate analgesics, and corticosteroids have all been reported to relieve pain from SOD attacks to varying degrees. ${ }^{57}$ Non-steroidal anti-inflammatory drugs were reportedly ineffective. ${ }^{6}$ Pancreatic enzyme supplementation has been reported to reduce dyspeptic symptoms, but have no effect on pain. ${ }^{5}$ Of note, Cheon et al. recently conducted a randomized controlled trial involving cases of suspected SOD: the administration of vardenafil into the duodenum distal to the papilla caused a significant decrease in basal sphincter of oddi pressures on SOM. ${ }^{58}$ This preliminary study included both cholecystectomized patients as well as those with intact gallbladders. More SOD-specific trials are needed to confirm the clinical potential of vardenafil in treating SOD. In summary, despite the paucity of data for the above treatments, given their relative safety and the benign course of PCS and SOD, experts recommend a trial of medical therapy prior to SOM and BSE.

Collective understanding of postcholecystectomy syndrome is still imperfect. Future studies are needed to elucidate the pathogenesis of SOD, to develop cost effective and non-invasive methods for evaluating SOD, and to pursue medical therapies as an adjunct or alternative to invasive interventions.

\section{References}

1. National Institutes of Health Consensus Development Conference. Statement on gallstones and laparoscopic cholecystectomy. Am J Surg 1993;165:390-8.

2. McPherson K, Wennberg JE, Hovind OB, et al. Small area variations in the use of common surgical procedures: an international comparison of New England, England, and Norway. N Eng J Med 1982;307:1310-4.

3. Ransohoff DF, Gracie WA. Treatment of gallstones. Ann Intern Med 1993;119:60619.

4. Finan KR, Ruth RW, Bryan MK, et al. Improvement in gastrointestinal symptoms and quality of life after cholecystectomy. Am J Surg 2006;192:196-202.

5 . Bodvall B. The post cholecystectomy syndrome. Clin Gastroenterol 1973;2:102-26.

6. Lasson A. The post-cholecystectomy syndrome: diagnostic and therapeutic strategy, Scand J Gastroenterol 1987;22:897-902.

7. Tondelli $\mathrm{P}$, Gyr K, et al. The biliary tract. Part I: Cholecystectomy. Clin Gastroenterol 1979;8:487-505.

8. Lasson A, Fork FT, et al. (1988). The postcholecystectomy syndrome: bile ducts as pain trigger zone. Scand J Gastroenterol 1988;23:265-71.

9. Filip M, Saftoiu A, et al. Postcholecystectomy syndrome - an algorithmic approach. J Gastrointestin Liver Dis 2009;18:67-71.

10. Baillie J. Sphincter of Oddi dysfunction. Curr Gastroenterol Rep 2010;12:130-4.

11. Jaunoo SS, Mohandas S, et al. Postcholecystectomy syndrome (PCS). Int J Surg 2010;8:15-7.

12. Bar-Meir S, Halpern Z, et al. Frequency of papillary dysfunction among cholecystec-

Figure 1. Diagnostic and therapeutic approach to postcholecystectomy syndrome. 
tomized patients. Hepatology 1984;4:32830 .

13. Elmi F, Silverman WB. Biliary sphincter of Oddi dysfunction type I versus occult biliary microlithiasis in post-cholecystectomy patients: are they both part of the same clinical entity? Dig Dis Sci 2010;55:842-6.

14. Geenen JE, Hogan WJ, Dodds WJ, et al. The efficacy of endoscopic sphincterotomy after cholecystectomy in patients with sphincter of Oddi dysfunction. N Engl $\mathrm{J}$ Med1989;320:82-7.

15. Madacsy L, Fejes R, et al. Characterization of functional biliary pain and dyspeptic symptoms in patients with sphincter of Oddi dysfunction: effect of papillotomy. World J Gastroenterol 2006;12:6850-6.

16. Desautels SG, Slivka A, et al. Postcholecystectomy pain syndrome: pathophysiology of abdominal pain in sphincter of Oddi type III. Gastroenterology 1999;116:900-5.

17. Kurucsai G, Joo I, et al. Somatosensory hypersensitivity in the referred pain area in patients with chronic biliary pain and a sphincter of Oddi dysfunction: new aspects of an almost forgotten pathogenetic mechanism. Am J Gastroenterol 2008;103:271725.

18. Hastbacka J, Jarvinen $\mathrm{H}$, et al. Results of sphincteroplasty in patients with spastic sphincter of Oddi. Predictive value of operative biliary manometry and provocation tests. Scand J Gastroenterol 1986;21:51620.

19. Linder JD, Klapow JC, et al. Incomplete response to endoscopic sphincterotomy in patients with sphincter of Oddi dysfunction: evidence for a chronic pain disorder. Am J Gastroenterol 2003;98:1738-43.

20. Toouli J. Sphincter of Oddi: Function, dysfunction, and its management. $\mathrm{J}$ Gastroenterol Hepatol 2009;24:S57-62.

21. Toouli J, Roberts-Thomson IC, et al. Manometry based randomised trial of endoscopic sphincterotomy for sphincter of Oddi dysfunction. Gut 2000;46:98-102.

22. Rolny P, Geenen JE, et al. Post-cholecystectomy patients with "objective signs" of partial bile outflow obstruction: clinical characteristics, sphincter of Oddi manometry findings, and results of therapy. Gastrointest Endosc 1993;39:778-81.

23. Thatcher BS, Sivak MV Jr, et al. Endoscopic sphincterotomy for suspected dysfunction of the sphincter of Oddi. Gastrointest Endosc 1987;33:91-5.

24. Corazziari E, Cicala M, et al. Scintigraphic assessment of SO dysfunction. Gut 2003;52:1655-6.

25. Sgouros SN, Pereira SP. Systematic review: sphincter of Oddi dysfunction-non-invasive diagnostic methods and long-term outcome after endoscopic sphincterotomy. Aliment Pharmacol Ther
2006;24:237-46.

26. Freeman ML, Guda NM. Prevention of post-ERCP pancreatitis: a comprehensive review. Gastrointest Endosc 2004;59:84564.

27. Freeman ML. Post-ERCP pancreatitis: patient and technique related risk factors. JOP 2002;3:169-76.

28. Madacsy L, Middelfart $\mathrm{HV}$, et al. Quantitative hepatobiliary scintigraphy and endoscopic sphincter of Oddi manometry in patients with suspected sphincter of Oddi dysfunction: assessment of flowpressure relationship in the biliary tract. Eur J Gastroenterol Hepatol 2000;12:77786.

29. Rome Foundation: Rome III diagnostic questionnaires. Available from: http:// www.romecriteria.org/questionnaires/

30. Steinberg WM, Salvato RF, et al. The morphine-prostigmin provocative test--is it useful for making clinical decisions? Gastroenterology 1980;78:728-31.

31. Corazziari E, Cicala M, et al. Hepatoduodenal bile transit in cholecystectomized subjects. Relationship with sphincter of Oddi function and diagnostic value. Dig Dis Sci 1994;39:1985-93.

32. Cicala M, Habib FI, et al. Outcome of endoscopic sphincterotomy in post cholecystectomy patients with sphincter of Oddi dysfunction as predicted by manometry and quantitative choledochoscintigraphy. Gut 2002;50:665-8.

33. Cicala M, Scopinaro F, Corazziari E, et al. Quantitative cholescintigraphy in the assessment of choledochoduodenal bile flow. Gastroenterology 1991;100:1106-13.

34. Craig AG, Peter D, et al. Scintigraphy versus manometry in patients with suspected biliary sphincter of Oddi dysfunction. Gut 2003;52:352-7.

35. Toouli J. Biliary scintigraphy versus sphincter of Oddi manometry in patients with post-cholecystectomy pain: is it time to disregard the scan? Curr Gastroenterol Rep 2005;7:154-9.

36. Thomas PD, Turner JG, et al. Use of (99m)Tc-DISIDA biliary scanning with morphine provocation for the detection of elevated sphincter of Oddi basal pressure. Gut 2000;46:838-41.

37. Matos C, Metens T, et al. Pancreatic duct: morphologic and functional evaluation with dynamic MR pancreatography after secretin stimulation. Radiology 1997;203: 435-41.

38. Aisen AM, Sherman S, et al. Comparison of secretin-stimulated magnetic resonance pancreatography and manometry results in patients with suspected sphincter of oddi dysfunction. Acad Radiol 2008;15:6019.

39. Pereira SP, Gillams A, et al. Prospective comparison of secretin-stimulated magnetic resonance cholangiopancreatography with manometry in the diagnosis of sphincter of Oddi dysfunction types II and III. Gut 2007;56:809-13.

40. Kalaitzakis E, Ambrose T, et al. Management of patients with biliary sphincter of Oddi disorder without sphincter of Oddi manometry. BMC Gastroenterol 2010;10:124.

41. Ros E, Navarro S, et al. Occult microlithiasis in idiopathic acute pancreatitis: prevention of relapses by cholecystectomy or ursodeoxycholic acid therapy. Gastro enterology 1991;101:1701-9.

42. Saraswat VA, Sharma BC, et al. Biliary microlithiasis in patients with idiopathic acute pancreatitis and unexplained biliary pain: response to therapy. J Gastroenterol Hepatol 2004;19:1206-11.

43. Parasher VK, Roman K, Sukumar R, et al. Is microlithiasis common in postcholecystectomy patients presenting with biliary type pain? Am J Gastroenterol 1996;91: 1939.

44. Quallich LG, Stern MA, et al. Bile duct crystals do not contribute to sphincter of Oddi dysfunction(or pain). Gastrointest Endosc 2002;55:163-6.

45. Okoro N, Patel A, et al. Ursodeoxycholic acid treatment for patients with postcholecystectomy pain and bile microlithiasis. Gastrointest Endosc 2008;68:69-74.

46. Jonson G, Nilsson DM, et al. Cystic duct remnants and biliary symptoms after cholecystectomy. A randomised comparison of two operative techniques. Eur J Surg 1991;157:583-6.

47. Walsh RM, Ponsky JL, et al. Retained gallbladder/cystic duct remnant calculi as a cause of postcholecystectomy pain. Surg Endosc 2002;16:981-4.

48. Topazian M, Salem RR, et al. Painful cystic duct remnant diagnosed by endoscopic ultrasound. Am J Gastroenterol 2005;100: 491-5.

49. Palanivelu C, Rangarajan M, et al. Laparoscopic management of remnant cystic duct calculi: a retrospective study. Ann R Coll Surg Engl 2009;91:25-9.

50. Shaw C, O'Hanlon DM, et al. Cystic duct remnant and the post-cholecystectomy syndrome. Hepatogastroenterology 2004; 51:36-8.

51. Bodner E, Aufschnaiter M, et al. Neuroma of the cystic nerve stump. No explanation for post-cholecystectomy pain. Chirurg 1978;49:424-7.

52. Iannelli A, Fabiani P, et al. Traumatic neuroma of the cystic duct with biliary obstruction. Report of a case. Acta Gastroenterol Belg 2003;66:28-9.

53. Nagafuchi Y, Katuki M, et al. A traumatic neuroma associated with obstructive jaun- 
dice after laparoscopic cholecystectomy. Hepatogastroenterology 1998;45:424-7.

54. Sand J, Nordback I, et al. Nifedipine for suspected type II sphincter of Oddi dyskinesia. Am J Gastroenterol 1993;88:530-5.

55. Khuroo MS, Zargar SA, et al. Efficacy of nifedipine therapy in patients with sphincter of Oddi dysfunction: a prospective, dou- ble-blind, randomized, placebo-controlled, cross over trial. Br J Clin Pharmacol 1992;33:477-85.

56. Fazel A, Li SC, et al. Octreotide relaxes the hypertensive sphincter of Oddi: pathophysiological and therapeutic implications. Am J Gastroenterol 2002;97:612-6.

57. Bar-Meir S, Halpern Z, et al. Nitrate thera- py in a patient with papillary dysfunction." Am J Gastroenterol 1983;78:94-5.

58. Cheon YK, Cho YD, et al. Effects of vardenafil, a phosphodiesterase type-5 inhibitor, on sphincter of Oddi motility in patients with suspected biliary sphincter of Oddi dysfunction. Gastrointest Endosc 2009;69:1111-6. 